\title{
Management of paediatric ocular inflammatory disease in the UK: national survey of practice
}

\author{
A. L. Solebo $0^{1,2,3}$ J. S. Rahi ${ }^{1,2,3}$ • C. Edelsten ${ }^{1,2}$ • J. L. Ashworth ${ }^{4,5}$. \\ A. D. Dick $3,6,7,8$ on behalf of the Paediatric Ocular Inflammation Group
}

Received: 25 October 2018 / Revised: 23 April 2019 / Accepted: 10 June 2019 / Published online: 9 July 2019

(c) The Royal College of Ophthalmologists 2019

Eye and child health specialists have recently come together to form the paediatric ocular inflammation group (POIG) [1]. POIG aims to inform management strategies, service commissioning and service delivery for children with sight threatening ocular inflammatory disorders. These disorders are united by their individual rarity, their complexity, and the use of potentially life-limiting systemic agents.

Using an online survey distributed through the Royal College of Ophthalmologists (Supplementary File), POIG sought to identify ophthalmic consultants routinely managing childhood uveitis or inflammatory ocular surface, orbital or optic nerve disorders. Of the 44 respondents, a third (17/44,

Supplementary information The online version of this article (https:// doi.org/10.1038/s41433-019-0518-8) contains supplementary material, which is available to authorised users.

A. L. Solebo

a.solebo@ucl.ac.uk

1 National Institute for Health Research Biomedical Research Centre at UCL Great Ormond Street Institute of Child Health and Great Ormond Street Hospital, London, UK

2 Great Ormond Street Hospital for Children NHS Trust, London, UK

3 National Institute for Health Research Biomedical Research Centre at Moorfields Eye Hospital NHS Foundation Trust and UCL Institute of Ophthalmology, London, UK

4 Division of Pharmacy and Optometry, School of Health Sciences, Faculty of Biology, Medicine and Health, The University of Manchester, Manchester, UK

5 Manchester Academic Health Science Centre, Manchester Royal Eye Hospital, Manchester, UK

6 Bristol Eye Hospital, University Hospitals Bristol NHS Foundation Trust, Bristol, UK

7 Pathology Department, Bristol Royal Infirmary, University Hospitals Bristol NHS Foundation Trust, Bristol, UK

8 Translational Health Sciences, Faculty of Health Sciences, University of Bristol, Bristol, UK
$38 \%$ ) managed all of these disorders (Fig. 1). Several specialists also managed adult disease: 14 of the $41(46 \%)$ managing childhood uveitis; 10/27 (38\%) orbital inflammation; 11/28 (39\%) inflammatory ocular surface disorders (OSD); and 12 of the 22 (56\%) ophthalmologists managing childhood optic neuritis. The majority had prescribed systemic immunomodulation/immunosuppression therapies: 30/41 in uveitis, $17 / 27$ in orbital inflammation (idiopathic orbital $n=10$, vasculitis related $n=4$, sarcoidosis $n=2$, and Behcet disease $n=1$ ), 6/28 OSD (blepharitic keratoconjunctivitis, KC $n=4$, atopic $\mathrm{KC} n=2$, herpetic keratitis $n=1$ ), and 14/22 in optic neuritis. Of those using systemic therapies, the majority did so as part of a multidisciplinary team: 20/30 in uveitis, $15 / 17$ in orbital inflammation, 14/14 in optic nerve disorders, and 5/6 OSD. Biological immunomodulation therapies had been prescribed (through rheumatology colleagues) by 28 specialists managing childhood uveitis.

Multidisciplinary care was also reported in the surveillance of children at risk of uveitis due to juvenile idiopathic arthritis. Examinations were carried out by allied health professionals in 12/38 (32\%) settings (optometrists $n=7$, orthoptists $n=5$, nurses $n=1$ ).

In summary, the majority of responding clinicians managed more than one disorder, and often as part of a multidisciplinary team. Approximately half also manage adults. The survey was "opt-in" in design, which may have resulted in under-ascertainment. However, an earlier national survey undertaken through the College identified a similar number (47) of UK surgeons managing congenital and infantile cataract [2], a disorder which has an incidence of 3-4 per 10,000 , thus 10 -fold more common than childhood uveitis, which has a reported UK incidence of 5 per 100,000 [3]. Nevertheless, there may have been under-ascertainment of clinicians managing mild disease.

Our findings suggest that half of all children with rare inflammatory eye disease are under specialists who do not manage adult disease. As the prognosis improves for children with rare eye disease, greater numbers will survive into 


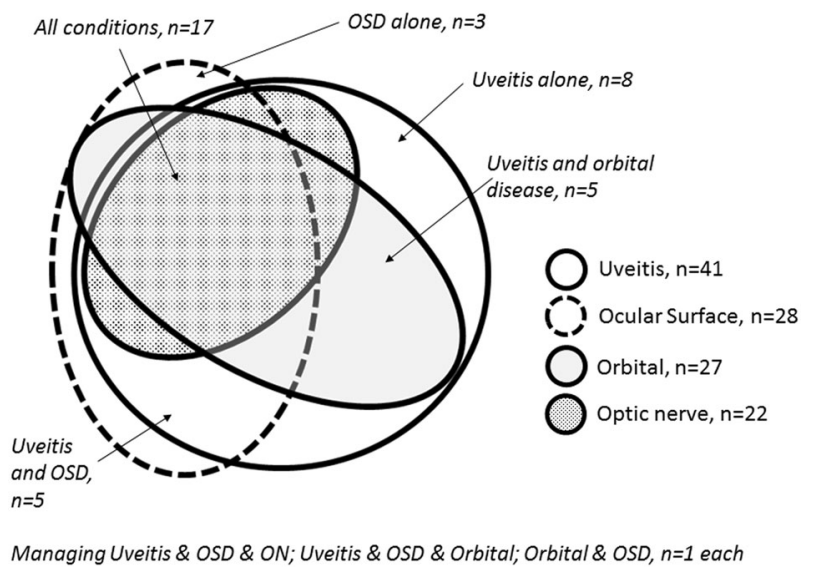

Fig. 1 Childhood ocular inflammatory disorders managed by survey respondents

adulthood with good vision. Effective transition processes are needed to prevent patient disengagement from care [4]. This is particularly important when care is shared between different services. We currently lack a consensus on best practice for transitional care for this group.

Collaborative national networks for rare disease have been recognised as a research priority by NHS England, particularly those networks which work to improve rare disease outcomes [5]. POIG has identified key clinicians managing these disorders (Fig. 1), and aims to develop the evidence base necessary to improve outcomes for individuals with (or at risk of) rare, childhood onset ocular inflammatory disorders.

Funding ALS and JSR are supported by the National Institute for Health Research (NIHR) Biomedical Research Centres at
Moorfields Eye Hospital/UCL Institute of Ophthalmology, and at the UCL Institute of Child Health/Great Ormond Street Hospital. JSR is a National Institute for Health Research (NIHR) Senior Investigator. ALS holds an NIHR Clinician Scientist award. Views expressed are those of the authors and not necessarily those of the NHS/NIHR.

\section{Compliance with ethical standards}

Conflict of interest ADD has received consultancy fees from Abbvie, Sanofi, Gyroscope, Roche. JLA has received consultancy fees from Abbvie. Remaining authors declare that they have no conflict of interest.

Publisher's note: Springer Nature remains neutral with regard to jurisdictional claims in published maps and institutional affiliations.

\section{References}

1. Solebo AL, Rahi JS, Dick AD, Ramanan AV, Ashworth J, Edelsten $\mathrm{C}$, and the POIG Uveitis Group. Areas of agreement in the management of childhood non-infectious chronic anterior uveitis in the UK. Br J Ophthalmol. 2019. pii: bjophthalmol-2018-313789. https://doi.org/10.1136/bjophthalmol-2018-313789.

2. Solebo AL, Russell-Eggitt I, Nischal KK, et al. Cataract surgery and primary intraocular lens implantation in children $<$ or $=2$ years old in the UK and Ireland: finding of national surveys. BJO. 2009;93:1495-8.

3. Edelsten C, Reddy MA, Stanford MR, et al. Visual loss associated with pediatric uveitis in English primary and referral centers. AJO. 2003; 135:676-80.

4. Stam H, Hartman EE, Deurloo JA, et al. Young adult patients with a history of pediatric disease: impact on course of life and transition into adulthood. J Adolesc Health. 2006;39:4-13.

5. Department of Health and Social Care. Implementation Plan for the UK Strategy for Rare Diseases. https://www.gov.uk/government/ publications/uk-strategy-for-rare-diseases-implementation-plan-forengland; 2018. Accessed March 2019. 